\title{
INVESTIGACIONES
}

\section{Desempeño académico en estudiantes de educación superior con Trastorno por Déficit de Atención}

\author{
Academic performance in higher education students \\ with Attention Deficit Disorder
}

\author{
Valeria Jimena Sepúlveda Bernales ${ }^{a}$, Valeria Fernanda Espina Araneda ${ }^{b}$ \\ ${ }^{a}$ Escuela de Terapia Ocupacional, Facultad de Salud, Universidad Santo Tomás, Chile. \\ valeria.sepulvedab@gmail.com \\ ${ }^{b}$ Escuela de Fonoaudiología, Facultad de Ciencias de la Salud, Universidad Autónoma de Chile, Chile. \\ valeria.espinaa@gmail.com
}

\begin{abstract}
RESUMEN
El Trastorno por Déficit de Atención e hiperactividad (TDAH) es un cuadro de origen neurobiológico, de alto impacto en la funcionalidad de las personas que lo padecen por sus alteraciones en la atención, la función ejecutiva y la motivación. El objetivo de esta investigación es conocer si existe relación entre el Trastorno por déficit atencional del adulto y el desempeño académico de estudiantes de educación superior.

Para esto se realizó una revisión bibliográfica y se concluyó que existe relación entre el TDAH y el desempeño académico. La revisión arrojó relación de estudiantes con TDAH y peor rendimiento, deserción universitaria y mayores cambios de carrera. Junto con esto, una alta comorbilidad, alteraciones psicoemocionales, sociales, alteraciones del sueño, tabaquismo y fatiga.

El desempeño de los estudiantes universitarios con TDAH se ve influenciado por las características del contexto y puede ser beneficiadas por estrategias neurodidácticas que consideren las dificultades expuestas.
\end{abstract}

Palabras claves: TDAH, estudiantes universitarios, Déficit atencional.

\begin{abstract}
The attention deficit hyperactivity disorder (ADHD) in adults has a neurobiological origin, with a high impact on the functionality of people who suffer from it due to alterations in attention, executive function and motivation. The objective of this research is to know if there is a relationship between the Attention Deficit Disorder of the adult and the academic performance of higher education students.

For this, a literature review was carried out and it concluded that there is a relationship between ADHD and academic performance. The review showed a relationship of students with ADHD and worse performance, university dropout and major career changes. Along with this, it showed a high comorbidity, psycho-emotional and social alterations, sleep disturbances, smoking and fatigue.

The performance of university students with ADHD is influenced by the characteristics of the context and can be benefited by neurodidactic strategies that consider the difficulties exposed.
\end{abstract}

Key words: ADHD, university students, attention deficit. 


\section{INTRODUCCIÓN}

En los últimos años, la aplicación de nuevas políticas públicas referentes a la educación superior ha promovido fuertemente el acceso de la población a la universidad y como consecuencia un aumento explosivo de oferta académica, teniendo como resultado un incremento considerable de jóvenes universitarios heterogéneos, diversos y con necesidades educativas individuales que obligan a las instituciones a fortalecer y crear diferentes estrategias de enseñanza y al mismo tiempo, considerar todas las características y dificultades que pueden estar presentes en la masificación de la educación superior (Fukushi, 2018).

Junto con el nuevo escenario de la educación superior en Chile, hoy se reconoce la necesidad de una didáctica centrada en el sujeto que aprende (Montes de Oca y Machado, 2011), por lo cual es de importancia reconocer el Trastorno por Déficit Atencional como una condición de alta prevalencia en el adulto, muchas veces subdiagnosticado (Asherson, 2012), y que tiene implicancias en el desarrollo de habilidades cognitivas de orden superior.

El trastorno por déficit atencional (TDAH) es una condición clínicamente heterogénea que está determinada por tres grupos de síntomas: la desatención, la impulsividad y la hiperactividad. Este trastorno ha sido ampliamente estudiado en la población infantil; sin embargo, sólo a partir del año 1976 se validó como diagnóstico en el adulto (Ticas y Ochoa, 2010). La prevalencia del trastorno se acerca al 5\% este grupo etario (Willcutt, 2012), en el cual está asociado a múltiples comorbilidades impactando en la participación social, personal y funcional (Jansen et al., 2012).

Las manifestaciones del trastorno presentan una gran variabilidad entre quienes lo padecen y se han relacionado con distintas alteraciones neurobiológicas, por ejemplo, alteraciones en los circuitos frontoestriatales y mesolímbicos (Cunill y Castells, 2015). El circuito frontoestriatal, está relacionado con las funciones ejecutivas y el mesolímbico con la transmisión de la dopamina. Se ha visto también que suelen rendir mal en tareas que evalúan estas funciones, especialmente la inhibición de respuestas no pertinentes, la planificación y la memoria de trabajo (Slachevsky et al., 2012).

La clínica del trastorno se sustenta en la neurobiología que la explica, la cual es amplia y diversa (Kasparek, Theiner y Filova, 2013) y en el adulto sugiere diversas problemáticas que impactan el funcionamiento de las personas que lo padecen (Colomer, Mercader, Berenguer, Roselló y Miranda, 2015). Junto con esto se le asocia a una alta comorbilidad y a características heterogéneas en los distintos individuos (Jansen et al., 2012). Si bien estos elementos han sido estudiados, no han sido ampliamente relacionados con estudiantes de educación superior lo cual se hace necesario para establecer las posibles consecuencias que podría tener el TDAH en el desempeño académico, por lo cual esta revisión tiene por objetivo conocer si existe relación entre las alteraciones del TDAH y el desempeño académico de estudiantes de educación superior.

\section{METODOLOGÍA}

Esta investigación se enmarca en la revisión bibliográfica del tipo descriptiva del TDAH en el adulto y su posible relación con el rendimiento académico en educación superior (Vera Carrasco, 2009). 
Para llevar a cabo la revisión, se seleccionaron fuentes secundarias de información el fin de acceder a bases de datos las cuales fueron: Proquest, EBSCO y ScienceDirect. Para la búsqueda de información se seleccionaron palabras claves relacionadas con el objetivo de la revisión. Las palabras utilizadas como concepto principal fue Trastorno por Déficit Atencional (TDAH), combinada con las variables neurobiología, adultos, perfil neuropsicológico, funciones ejecutivas, universitarios, educación superior y aprendizaje, incluyendo resultados en idioma español e inglés.

Para realizar la búsqueda se usaron los siguientes criterios de inclusión,

- Artículos con un máximo de 10 años de antigüedad.

- Artículos que contuviera al menos una combinación de TDAH y otra palabra clave.

- Artículos que pertenecieran exclusivamente a las fuentes seleccionadas.

Se utilizaron los siguientes criterios de exclusión

- Artículos relacionados con efectividad de tratamientos (farmacológicos, psicológicos u otros).

- Artículos relacionados con TDAH en la infancia.

El periodo de búsqueda se realizó entre septiembre y noviembre del año 2018 y se encontró un total de 2580 artículos, de los cuales se seleccionaron 118 utilizando los criterios de exclusión e inclusión y atingencia con el objetivo central de la revisión. Estos se clasificaron en las temáticas iniciales y emergentes las cuales fueron: aspectos generales, aprendizaje en estudiantes de educación superior, neurobiología del trastorno y perfil neuropsicológico.

A partir de esta revisión se interpretaron los datos y se organizó la información desde lo más general a lo específico.

\section{RESULTADOS}

\subsection{ASPECTOS GENERALES DEL TDAH}

El trastorno por déficit atencional (TDAH) es una condición que tiene inicio en la infancia, pero la mayoría de los sujetos que la padecen la mantienen en la adultez. El TDAH se presenta con tres grupos de síntomas: inatención, impulsividad, hiperactividad (Mayor y García, 2011).

La última edición del Manual Diagnóstico y Estadístico de los Trastornos Mentales (DSM-V), lo clasifica dentro del grupo de Trastornos del Desarrollo Neurológico, y lo define como un patrón persistente de inatención (mayor a 6 meses) y/o hiperactividad. La inatención se caracteriza por dificultad de poner atención en los detalles, mantener la atención en tareas o actividades recreativas, parecer no estar escuchando, no seguir instrucciones y no terminar las tareas, dificultad para organizar tareas y actividades, dificultad para iniciar tareas que requieran esfuerzo sostenido, pierde cosas, se distrae con estímulos externos y olvida las actividades cotidianas. Para el diagnóstico en niños se requieren 6 de los puntos anteriores. 
La hiperactividad, según el DSM-V, se caracteriza por, juguetear o golpear manos o pies, no permanece sentado, trepa en situaciones inapropiadas, incapaz de jugar tranquilamente, actúa como si lo impulsara un motor, habla excesivamente, responde antes que termine la pregunta, le es difícil esperar su turno, irrumpe o se inmiscuye con otros. Los síntomas deben interferir con el funcionamiento y deben estar presentes en dos o más contextos.

El manual no hace una clasificación distinta para adultos, sin embargo, especifica ciertas diferencias: a. se requieren cinco en vez de seis criterios para el diagnóstico; b. especifica tareas de esfuerzo sostenido en adultos como informes, formularios o largos artículos; c. la distracción de estímulos externos podría ser por pensamientos no relacionados; y d. actividades cotidianas cómo devolver llamadas, pagar facturas, acudir a las citas.

Una de sus características principales es la heterogeneidad que subyace al cuadro, tanto en la sintomatología inter-sujetos como intra-sujeto, mostrando variabilidad de conductas entre el grupo que padece TDAH como en las respuestas fluctuantes que evidencia un mismo sujeto dependiendo de factores como la hora del día, siendo más pobres los rendimientos por la tarde que por la mañana; evidenciando cambios en la conducta dependiendo del tipo de tarea, el contexto y la motivación (López, Rodillo y Kleinsteuber, 2008).

En relación a la sintomatología, en la adultez, el principal cambio es en la hiperactividad, la cual disminuye o pasa a presentarse como inquietud interna. Se mantiene la inatención y la impulsividad, que se evidencia en pérdida de tolerancia a la frustración o impaciencia. Las personas que presentan TDAH tienen menores logros académicos que las personas que no lo tienen, a pesar de tener igual inteligencia, lo cual se traduce posteriormente en menores ingresos económicos (Quintero et al., 2011). De igual forma, el trastorno no sería un problema en el conocimiento si no en el rendimiento (Barkley, Murphy y Fischer, 2010).

Una de las explicaciones del trastorno en la etapa adulta, sugiere que no siempre las alteraciones ejecutivas serían centrales, sino que, en algunos casos primaría lo entendido como "aversión a la espera", lo cual los hace preferir las recompensas inmediatas por sobre las que podrían tener un beneficio mayor. Esto corroborado con neuroimágenes que muestran hipoactivación en el sistema estriado ventral, responsable del sistema de recompensa cerebral (Slachevsky et al., 2012). En relación a esto, otros artículos incluyen en este defecto la participación del circuito mesolímbico que podría explicar las dificultades motivacionales (López-Martín et al., 2010).

Las dificultades en los contextos sociales académicos y problemas conductuales que aparecen en la infancia se convierten en peor adaptación laboral y mayor riesgo de ser despedidos. Se le suma mayor incidencia de divorcios, dificultades de pareja e interpersonales, embarazos no deseados, conductas antisociales y accidentes de tránsito (Quintero et al., 2011).

Si bien se sabe que los síntomas se atenúan al aumentar la edad, muchos adultos con TDAH compensan sus dificultades eligiendo estilos de vida y carreras que se ajusten a sus dificultades y aunque parecen funcionar bien, lo realizan a costa de un gran gasto de energía (Schoeman, Albertyn y de Klerk, 2017).

La prevalencia de este diagnóstico en adultos es algo controversial, mientras algunos investigadores afirman su infradiagnóstico (Asherson, 2012), existe también evidencia de lo contrario (Paris, Bath y Thombs, 2015). Se estima que la población adulta con TDAH alcanza entre un 4 y 7\%, y que no varía en distintas zonas geográficas, cultura o nivel socioeconómico. Sin embargo, en poblaciones especiales como personas con abuso o 
dependencia a drogas llegaría al 20\% y en población penitenciaria al 25\%. Las diferencias por género disminuyen significativamente en la adultez (Ticas y Ochoa, 2010).

Estudios han encontrado que el TDAH en adultos puede existir en el $60 \%$ de personas que iniciaron sus síntomas antes de los siete años (Valdizán e Izaguerri-Gracia, 2009).

En relación a estudiantes de educación superior, existe escasa información acerca de su prevalencia, sin embargo, un estudio realizado a estudiantes de medicina en Chile obtuvo una prevalencia del TDAH en un 30\%. (Santander, Berner, Contreras y Gómez, 2013).

\subsection{BASES NEUROBIOLÓGICAS DEL TDAH}

La neurobiología del TDAH no responde a un área específica del cerebro ni a una falla específica, sino que, a variadas alteraciones en vías y redes cerebrales, factores hereditarios, actividad metabólica, estructural y fisiológica (Aboitz et al., 2012).

Los factores hereditarios podrían representar el $80 \%$ de los casos diagnosticados con TDAH (Portela Sabari et al., 2016). Por otro lado, estudios de neuroimagen demuestran que podrían existir anomalías estructurales en regiones que forman el circuito fronto-estriado, corteza prefrontal dorsolateral y regiones dorsales de la corteza cingulada anterior y el estriado, ganglios basales y los lóbulos frontales. Además, se ha encontrado una reducción del cuerpo calloso, núcleo caudado y cerebelo (Portela Sabari et al., 2016).

El metabolismo hace referencia a los procesos que se dan en el cerebro, a través del oxígeno y la glucosa, y que permiten obtener la energía necesaria para el procesamiento de la información. El tejido cerebral tiene mucha actividad eléctrica y requiere altos niveles de energía, por lo cual el funcionamiento metabólico es esencial. En personas con TDAH se ha observado reducción del metabolismo en el lóbulo frontal, corteza parietal, núcleo caudado y cerebelo. Por otro lado, se ha visto aumento del flujo sanguíneo y actividad eléctrica en la corteza sensorio-motora (Portela Sabari, 2016).

Otro aspecto importante en el funcionamiento cerebral es la dinámica que ocurre con los neurotransmisores en las distintas estructuras cerebrales. Se ha relacionado el TDAH con la alteración en los procesos implicados en la transmisión de dopamina, influyendo en la conducta orientada a metas, función inhibitoria, predicción de eventos, aversión a la espera, atención, inquietud y motivación (Aboitiz et al., 2012; Volkow et al., 2010; Plichta et al., 2009).

Se ha visto también alteraciones de la Serotonina, involucrada en el ánimo y Acetilcolina (Aboitiz et al., 2012), relacionada con la mantención de la alerta, que es el punto de partida de la atención, y podría estar relacionada con el consumo de tabaco en adolescentes y adultos con TDAH (Castillo, 2015).

Por otra parte, se han encontrado alteraciones en la conectividad en distintas zonas cerebrales, se ha visto baja conectividad de las redes fronto-estriatal, corteza prefrontal dorsolateral y ventrolateral, corteza orbitofrontal, las regiones parietales superiores, el núcleo caudado, el tálamo, la amígdala y el cerebelo (Kasparek, Theiner y Filova, 2013; Portela Sabari et al., 2016) y alteraciones en los patrones de conectividad interregional funcional (Rodillo, 2015).

En adultos con TDAH, se ha observado que tienen en general una desregulación de la activación cerebral o arousal, esto lleva a tener menos estados de vigilancia y más de somnolencia e inicio del sueño (Straub et al., 2018), además en tareas cortas de atención utilizan menos recursos corticales que personas que no presentan TDAH (Hasler et al., 2016). 


\subsection{PERFIL NEUROPSICOLÓGICO DEL TDAH}

Por definición, la atención es la principal dificultad en personas con TDAH; en adultos, se observan dificultades en regular el nivel de atención según la demanda de la tarea y dificultades en el procesamiento de estímulos irrelevantes. (Salmi et al., 2018). Sin embargo, también se ha observado dificultad en el procesamiento voluntario de atención y en tareas que requieren resolución de conflictos (Hasler et al., 2016). En definitiva, todo el sistema atencional estaría alterado y los adultos presentarían dificultades en el nivel de alerta, en responder a un estímulo relevante y en la atención voluntaria.

Se ha planteado también que las personas que tienen TDAH del subtipo inatento tendrían dificultad en la rapidez del procesamiento de la información y en la atención selectiva, mientras que los que se presentan con el subtipo combinado se afecta la capacidad para mantener la atención y considerar sólo los estímulos relevantes en una tarea (Mellado, Martínez y Tello, 2013).

Junto con la atención, las alteraciones ejecutivas son un déficit central en el TDAH y diversas investigaciones apoyan la teoría que las características clínicas del trastorno responden a una alteración de las funciones ejecutivas (Ramos y Pérez, 2015).

Las afectaciones ejecutivas que se han descrito alteradas para el TDAH son autorregulación del comportamiento, desinhibición, impulsividad, dificultades en el control de respuestas automáticas, hiperactividad y dificultades en la regulación de emociones (Ramos y Pérez, 2015). Estudios han demostrado también, dificultades en la memoria de trabajo, monitorización, flexibilidad, control atencional y planificación (Ramos y Pérez, 2015).

Como se ha explicado anteriormente, los adultos con TDAH presentan diferentes dificultades cognitivas, sin embargo, la literatura sugiere que existe un área que se encuentra fortalecida, la creatividad. Un estudio realizado a estudiantes universitarios de la Universidad de Memphis demostró que aquellos que tenían TDAH respondían mejor ante una prueba que requería de pensamiento innovador, y con una distancia semántica mayor que los controles. Esto se correlacionaría con estudios que demuestran que las dificultades atencionales podrían derivar en un mejor pensamiento divergente (White y Shah, 2016).

Por otro lado, entender la hiperactividad y la búsqueda de sensaciones como conductas autorreguladoras de un cerebro hipoactivo en la regulación de la excitación podría interpretarse como una compensación para la autorregulación de este cerebro inestable (Strauß et al., 2018).

\subsection{APRENDIZAJE EN ESTUDIANTES DE EDUCACIÓN SUPERIOR CON TDAH}

El aprendizaje en adultos con TDAH, por las características cognitivas mencionadas anteriormente, puede verse influida positiva o negativamente por distintos factores. En el contexto de la educación superior actual hay que comprender que esta ha vivido un proceso de democratización que ha llevado consigo la incorporación de muchos más jóvenes a las aulas, que antes no tenían acceso a este nivel educativo. Este proceso ha ocurrido rápidamente y como consecuencia, las clases se desarrollan en aulas sobrepobladas, heterogéneas y diversas, esto dificulta la didáctica docente, que debe privilegiar clases expositivas, métodos convencionales y menos personalización de la enseñanza (Olivos, 2011). 
En Chile, el alumnado actual se caracteriza por pertenecer en un gran porcentaje a la clase media emergente, ven la educación superior como una oportunidad, no son receptores pasivos, y exigen métodos pedagógicos que consideren su contexto sociocultural (Fukushi, 2010). Por otra parte, la diversidad del alumnado exige métodos menos estandarizados, más heterogéneos y flexibles, que requieren de formación continua del profesorado. Junto con esto, la fragmentación del currículum implica exigencias docentes de entregar gran cantidad de contenido, con metodologías altamente teóricas (Olivos, 2011).

La primera barrera para los estudiantes con TDAH es la transición a la vida universitaria, esta implica menor estructura en el entorno académico y niveles más bajos de supervisión de los padres y docentes (Prevatt y Young, 2014) lo cual los hace asumir autonomía y decisiones que en otras etapas no están presentes.

En relación al contexto en que se desenvuelve la persona, ya sea familiar, escolar o social, este va a influir en la severidad de los síntomas y el pronóstico del cuadro, y a su vez esto va a incidir en los procesos de autorregulación necesarias para la adaptación al contexto académico. Además, si el entorno de aprendizaje universitario no brinda herramientas, es factible que la evolución del trastorno sea negativa, profundice los síntomas y disminuye la calidad de vida, autoestima y desempeño académico y social. (Zuluaga-Valencia y Fandiño-Tabares, 2017).

El desempeño académico se puede entender como la relación entre variables ambientales, procesos de aprendizaje y enseñanza, y persistencia (York et al., 2015). Dentro de los procesos de aprendizaje se encuentran las variables cognitivas, sin embargo, estas no han sido ampliamente estudiadas en la educación superior (Rodríguez Portuguez, 2016).

Para abordar estos aspectos, es de relevancia considerar como la neurodidáctica puede aportar en el desarrollo de estrategias efectivas en estudiantes con TDAH.

La neurodidáctica es el área de la pedagogía que toma los aportes de las neurociencias para desarrollar estrategias didácticas para la educación (Paniagua, 2013). Para el aprendizaje de estudiantes con TDAH, particularmente por las implicancias neurobiológicas, se requiere revisar cómo se ven favorecidos por esta mirada.

Según lo mencionado en la descripción del trastorno, la variabilidad de respuesta intrasujeto implica seleccionar estrategias que consideren el perfil de los estudiantes, ya que se ha visto que la sintomatología puede variar según la hora del día, siendo los rendimientos más pobres por la tarde, las situaciones que tengan bajos estímulos, tareas que requieran habilidades de organización o de restricción de la actividad motora (López et al., 2008).

Uno de los aspectos estudiados es el uso de actividades intrínsecamente interesantes, especialmente donde la persona tiene talento, pueden facilitar la motivación. Junto con esto, las actividades con recompensa inmediata facilitan el involucramiento en tareas. Esto se refiere, por ejemplo, a evaluaciones con menor contenido, como controles o actividades de menor duración con retroalimentación a corto plazo (Volkow et al., 2010).

López-Martín, Albert, Fernández-Jaén y Carretié (2010) destacan los estudios de Luman, Oosterlann y Sergeant (2005) que plantean que las personas con TDAH dependen de un refuerzo externo mayor, más frecuente e inmediato que los grupos controles para obtener su máximo rendimiento.

En relación al uso de la tecnología, los adolescentes y adultos jóvenes tienen habilidades para el manejo y selección de contenidos audiovisuales en pantallas en paralelo, pero esto sugiere un riesgo de que la saturación informativa les impida comprender los mensajes. Por esta razón es importante la mediación y el uso de estas herramientas para lograr una 
reflexión consciente de lo observado, la discriminación de los estímulos y el aporte de la motivación en los procesos atencionales (Barandiarán y Samaniego, 2013).

Varios investigadores señalan que la cultura audiovisual actual (videojuegos, televisión, internet, etc.), promueven escasas oportunidades para favorecer y entrenar la atención sostenida, la latencia en la espera de recompensa, las acciones reflexivas, metacognición, etc.; en todos los individuos siendo, posiblemente, su impacto mayor en aquellos con TDAH (Barandiarán y Samaniego, 2013).

Los adultos jóvenes con TDAH se benefician menos de la información visual en presencia de ruido, por lo que las estrategias visuales que se utilicen deberían ir acompañadas de una posible regulación del ruido ambiente. Por otra parte, al destacar las palabras claves y aumentar el tiempo de respuesta supone un aporte para la comprensión de textos (Michalek et al., 2014).

En estudios de selección de contenidos televisivos, Barandiarán y Samaniego (2013) refieren que para los adolescentes con TDAH todos los tópicos relacionados con el humor llaman más su atención que para el grupo control y están dentro de sus preferidos a la hora de elegir en que focalizar su interés, estos datos podrían extrapolarse a quiebres comunicativos utilizados en la sala de clases para acercar los contenidos y activar procesos de atención sostenida.

Además de las estrategias que pueden llevarse a cabo en el aula, la evidencia sugiere la realización de programas de apoyo a los estudiantes con TDAH, especialmente con un enfoque psicoeducativo.

Se ha observado que las temáticas útiles son:

- Habilidades académicas: Estrategias de estudio, toma de notas, escritura de documentos.

- Atención: Estrategias para disminuir la distractibilidad, entrenamiento en Mindfulness.

- Cognición: identificar distorsiones cognitivas.

- Control emocional: trabajo de la frustración y emociones explosivas.

- Establecimiento de metas: a corto, mediano y largo plazo.

- Gestión de vida: ejercicio, nutrición, sueño, finanzas.

- Organización y planificación: uso de cajas, agenda, otros recursos.

- Resolución de problemas: desglose de tareas complejas.

- Psicoeducación: con relación al TDAH.

- Relaciones y comunicación: estrategias y afrontamiento.

- Autoestima y auto-refuerzo: trabajo en la confianza y refuerzos a corto plazo.

Estos tópicos debieran ir acompañados de tareas semanales donde se refuercen en contextos reales (Prevatt y Young, 2014).

El ingreso a la educación supone, por lo general, la realización de una prueba de admisión, la cual, puede ser de gran dificultad en estudiantes con TDAH, los que podrían favorecerse con tiempos extendidos o espacios con menor distracción para rendirla (Prevatt y Young, 2014). En Chile, el Departamento de Evaluación, Medición y Registro Educacional (Demre), que se encarga de la Prueba de Selección Universitaria (PSU), ofrece a los estudiantes que presentan este diagnóstico, solicitar adecuaciones en el tiempo, la sala, las pausas u otras que pueden ayudar a un mejor rendimiento (Demre, 2018). 
Figura 1. Aspectos que influyen el aprendizaje de estudiantes con TDAH

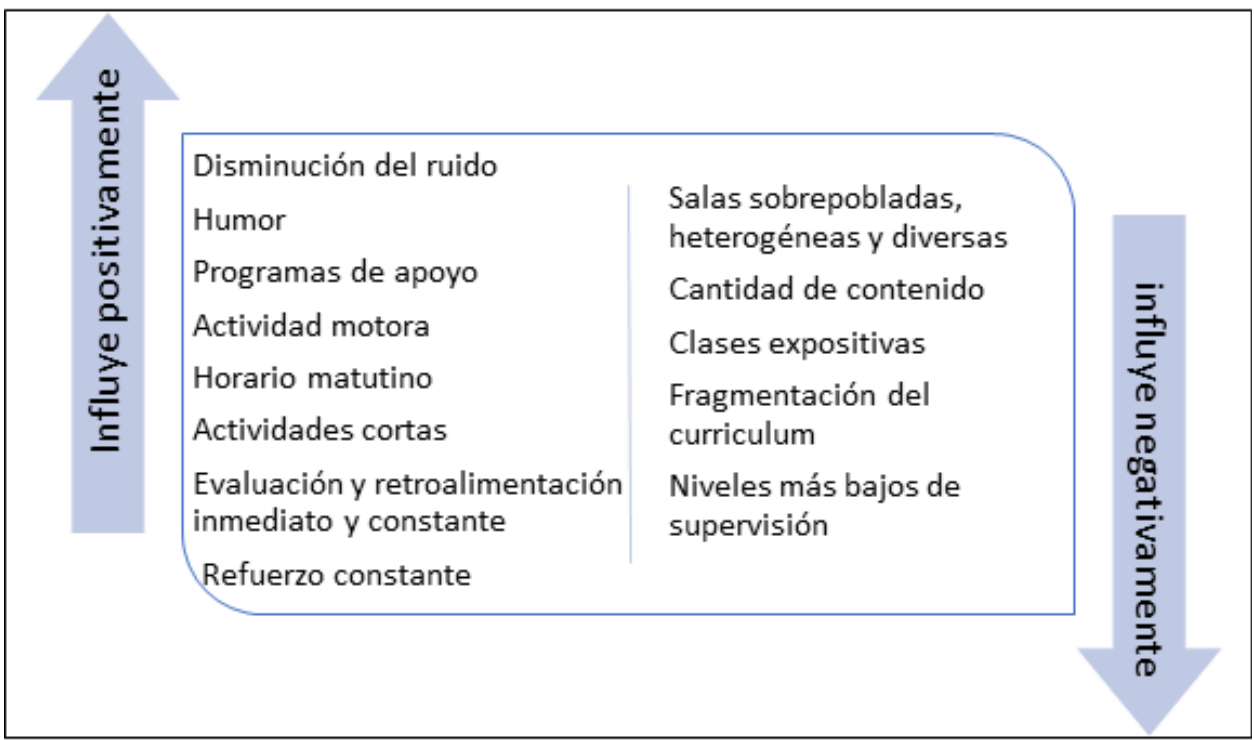

Fuente: Elaboración propia.

\subsection{CONSECUENCIAS DEL TDAH EN EL DESEMPEÑO DE ESTUDIANTES DE EDUCACION SUPERIOR}

La educación superior requiere que los estudiantes logren cumplir con un plan de estudios a través de la aprobación de las distintas asignaturas que lo componen. Para estudiantes con TDAH, aparece como una dificultad relevantes, en primer lugar, porque el TDAH en el adulto se identifica con un rendimiento menor que el esperado a la inteligencia y nivel educativo (Ortiz y Jaimes, 2016); además, estudiantes de educación superior con TDAH tienen peores calificaciones que el promedio, esto también podría relacionarse con estudios previos que sugieren que la memoria de trabajo se relaciona significativamente con el promedio de calificaciones (Prevatt y Young, 2014), y con que la función ejecutiva de planificación es un factor predictor de rendimiento académico (Said, 2013).

Un estudio longitudinal mostró que las personas que eran diagnosticadas en la infancia tenían peores resultados académicos y ocupacionales que el grupo control. Se observó también que tenían once veces más probabilidades de que al finalizar la educación secundaria, no se inscribieron en la educación superior o un mayor porcentaje lo hiciera en carreras más cortas (Kuriyan et al., 2013).

Por otro lado, se ha evidenciado que los estudiantes con TDAH presentan menores promedios, mayores cambios de carrera, más frecuencia de abandono y más tiempo en terminar la carrera (Ortiz y Jaimes, 2016).

En relación a la motivación, se han observado menores respuestas hemodinámicas relacionadas con la frecuencia cardiaca y coloración de la piel ante la recompensa, los sujetos con TDAH prefieren resultados rápidos, aunque la demora implique mejores y/o mayores desenlaces positivos, esto termina por propiciar la deserción frente a los proyectos. 
Esto se suma a que cuando se exponen a situaciones en las que no pueden disminuir los tiempos de espera de la recompensa "intentarán reducir la experiencia subjetiva de espera dirigiendo su atención a otros estímulos e incrementando el nivel de estimulación a través del movimiento" provocando comportamientos disruptivo y descontextualizado que afectarán en su autoconcepto y la participación social (López-Martín et al., 2010).

La autorregulación o autocontrol se hace más evidente en esta etapa, ya que deben asumir responsabilidad en lo personal y social, se manifiestan en conductas que interfieren como hablar continuamente, entrar y salir del aula, interrumpir clases y a sus compañeros, impuntualidad, desorganización, abandono a los estudios (Ortiz y Jaimes, 2016). También tienen dificultades para terminar las tareas o pruebas en el tiempo destinado y la percepción de trabajar más duro que sus compañeros (Prevatt y Young, 2014).

Estas manifestaciones podrían pasar desapercibidas por docentes de educación superior, dado que, a diferencia de la educación básica y media, las salas suelen ser más grandes, la asistencia no es obligatoria y al ser adultos la distracción puede ser menos visible (Prevatt y Young, 2014). Sin embargo, aparecen más evidentes dificultades en la administración del tiempo, insatisfacción con el rendimiento académico y las relaciones interpersonales (Kwon, Kim y Kwak, 2018).

La dificultad para planificar la acción no permite tomar las instrucciones y hacerlas propias de su plan de respuesta, haciendo difícil la ejecución adecuada de tareas tanto académicas como sociales, lo que generalmente tiende a desencadenar conductas de riesgo y altos niveles de accidentalidad y vulneración a terceros. (Zuluaga-Valencia y FandiñoTabares, 2017). Esto podría ser un factor influyente en la alta prevalencia del trastorno en población penal (Ticas y Ochoa, 2010).

La presencia de un déficit general en el procesamiento emocional podría explicarse por las alteraciones funcionales en el circuito prefrontal-amigdalar del cerebro, este está implicado en la evaluación del significado emocional de los acontecimientos, por lo cual las personas con TDAH responden de manera deficiente en resultados en tareas de reconocimiento emocional a partir de la expresión facial, la prosodia y el análisis contextual para interpretar la emoción de otros (López-Martín et al., 2010).

La posible implicación de la amígdala cerebral, mencionada anteriormente, podría ser la responsable de los defectos de comprensión emocional contextual y las correlaciones observadas en los niveles de ansiedad, depresión, excesiva reactividad y labilidad emocional mostrados por las personas con TDAH (López-Martín et al., 2010).

Por otro lado, estudiantes universitarios con TDAH evidencian relaciones amorosas de peor calidad. Particularmente, el nivel de síntomas en mujeres universitarias se relaciona negativamente con la calidad de la relación. Esto se explicaría a partir de las dificultades de regulación emocional, que además se presenta como un mediador de síntomas depresivos. Junto con esto, la calidad de las relaciones está influida por el estrés percibido y el conflicto en las relaciones hostiles (Bruner, Kuryluk y Whitton, 2015).

La comorbilidad del TDAH en el adulto, es decir la coexistencia temporal de dos o más trastornos psiquiátricos en la persona, afecta a tres de cada cuatro personas. Los adultos con TDAH tienen más riesgo de sufrir un trastorno grave de la personalidad, asî como de dependencia a substancias (Almeida Montes, Ricardo-Garcell, Prado Alcántara y Martínez García, 2009). Además, se ha visto comorbilidad con: trastornos del ánimo (19 al $37 \%$ ), trastornos ansiosos (25 a 50\%) en abuso de alcohol (32 a 53\%), otros tipos de abuso (8 a 32\%), trastornos de personalidad (10 a 20\%), conducta antisocial (18 a $28 \%$ ) 
(Valdizán y Izaguerri-Gracia, 2009). Cada una de estas alteraciones podrían tener efectos en el desempeño académico que se sumarían a, los ya mencionados, propios del TDAH.

El consumo y abuso de drogas, puede ser un factor de relevancia en el desempeño académico. Si bien se sabe que las consecuencias del consumo de drogas son perjudiciales, algunas drogas, incluida la anfetamina, la nicotina o el alcohol, tienen la capacidad, al igual que los reforzadores naturales, de aumentar la concentración de dopamina en el núcleo accumbens, activando el sistema de recompensa. En este sentido, el abuso de sustancias en el TDAH podría interpretarse como una forma de compensación o automedicación. (López et al., 2008). Por esto, la temática relacionada con las drogas es un eje necesario de abordar en estudiantes con TDAH.

Con relación al sueño, estudios han demostrado que los ciclos circadianos se encuentran alterado en cuanto a calidad y cantidad del sueño. (Baird, Coogan, Siddiqui, Donev y Thome, 2012).

Otro aspecto es la mayor adicción a la nicotina en adultos con TDAH, lo que se acompaña con mayores dificultades para dejar de fumar, dado la mayor intensidad de síntomas de abstinencia al intentarlo. (Kollins et al., 2013).

Un estudio realizado con adultos con TDAH reveló que presentan significativamente más fatiga, tanto física como mental. Estos niveles de fatiga se pueden asociar a un peor funcionamiento, además el $45 \%$ de los participantes con TDAH declaró haber tenido un periodo de fatiga que los hizo consultar al médico, en comparación con un $17 \%$ en el grupo control (Rogers, Dittner, Rimes y Chalder, 2017).

A esto se suma que se ha visto que los adultos con TDAH presentan menos continuidad de tratamiento en la edad adulta, que no se relaciona necesariamente con mejoría de la sintomatología (Prevatt y Young, 2014), esto sería negativo, ya que existe evidencia que sugiere que los mecanismos de control cognitivo se normalizan después del tratamiento con metilfenidato (Nielsen y Wiig, 2011). 
Figura 2. Consecuencias del TDAH en estudiantes de educación superior

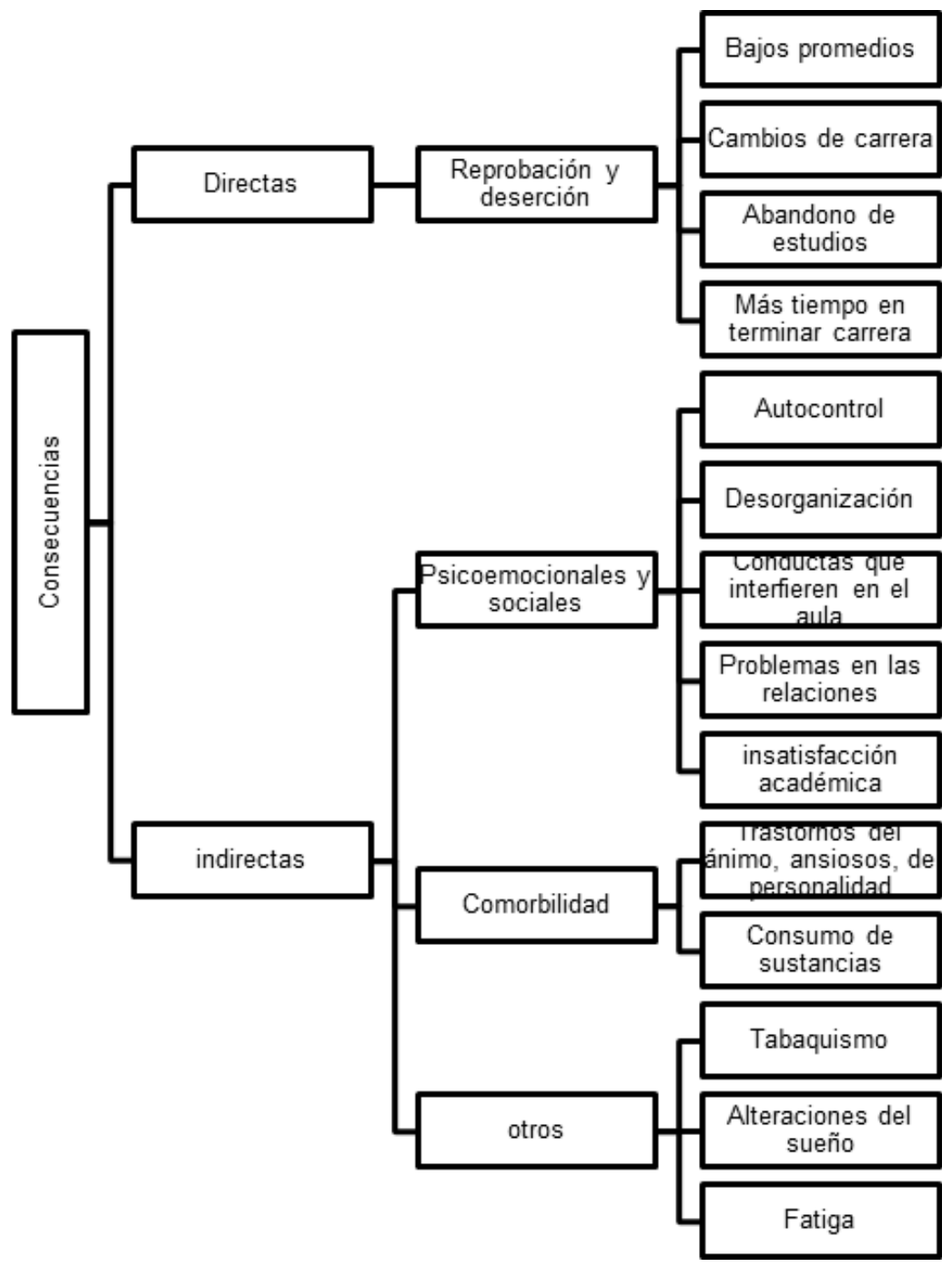

Fuente: Elaboración propia.

\section{DISCUSIÓN}

El TDAH es un trastorno de inicio en la infancia, pero que en la mayoría de los casos se mantiene en la edad adulta, se caracteriza por presentarse con grupos de síntomas como la inatención, la hiperactividad y la impulsividad. En el adulto toma características distintas que en la infancia y conlleva una serie de comorbilidades y riesgos (Ticas y Ochoa, 2010). Las dificultades presentes en estudiantes con TDAH, no solo a nivel cognitivo, podrían influir en su desempeño académico, lo que implica desarrollar nuevas estrategias o 
programas que permitan abordar el tema. Asimismo, el escenario actual de la educación superior sugiere la necesidad de considerar la diversidad y heterogeneidad del estudiantado.

A partir de la revisión bibliográfica realizada, es posible decir que existe relación entre las alteraciones del TDAH y el desempeño académico de estudiantes de educación superior. Esta relación se sustenta en el principio, ampliamente difundido en la bibliografía estudiada, que establece que el trastorno se explica por una disfunción tanto estructural como funcional de áreas cerebrales relacionadas con las funciones cognitivas necesarias para el aprendizaje, la motivación y la capacidad de resolver problemáticas asociadas al contexto universitario.

Existe acuerdo entre los autores acerca de la alta prevalencia (Thomas et al., 2015) que existe en la población general de TDAH y el aumento significativo en las últimas décadas del reconocimiento de la sintomatología en el adulto (Ticas y Ochoa, 2010). Por otro lado, llama la atención que específicamente en estudiantes de educación superior el estudio epidemiológico aún es incipiente.

La expresión de una genética hereditaria alterada podría corresponder al $80 \%$ de los casos con sintomatología clínica de TDAH, siendo interesantes los aportes obtenidos en estudios entre padres y hermanos (Portela Sabari et al., 2016) que manifiestan la relevancia de la heredabilidad en el cuadro.

El análisis crítico de la información recopilada concluye que no existe un modelo único y universal para explicar las bases neurobiológicas del TDAH y fortalece el enfoque de entender el cuadro desde un punto de vista multifactorial y heterogéneo, en el que están involucrados múltiples procesos fisiopatológicos subyacentes a la expresión de los síntomas.

Con relación al perfil neuropsicológico de los adultos con TDAH, las investigaciones han entregado información relevante y consistente con la alteración no solo de la atención, si no que acerca de la importancia que tiene la afectación de las funciones ejecutivas en el desempeño. Sin duda, y como se mencionó anteriormente, la heterogeneidad del cuadro dificulta establecer parámetros absolutos y la complejidad de los procesos involucrados supone un desafío constante en establecer métodos evaluativos que den cuenta de forma precisa de las alteraciones neuropsicológicas.

Otro aspecto relevante es lo que ocurre en relación a la motivación. En el estudio realizado se encontró que las dificultades asociadas al sistema de recompensa repercuten en las personas con TDAH, ya que requieren de resultados inmediatos para la mantención de la motivación. Se evidencia una frecuente aversión a la espera y eso implica que elijan tareas y métodos que otorgan menores beneficios.

La bibliografía es amplia en la descripción de los tipos de comorbilidades y su prevalencia. Existe acuerdo en la alta coexistencia de psicopatologías y cuáles son estos cuadros asociados al TDAH (Zuluaga-Valencia y Fandiño-Tabares, 2017), sin embargo, la bibliografía es pobre en el análisis de estas asociaciones y su vinculación con el desempeño académico.

Existen escasos estudios que vinculan el TDAH con las consecuencias en la educación superior y es una temática menos abordada que requiere investigaciones futuras más exhaustivas. No obstante, esta investigación permitió establecer las posibles implicancias que tiene el TDAH en el desempeño académico y que sugieren que la conjunción de estos elementos más los factores contextuales, personales y sociales que afectan a estudiantes con TDAH tendrían un impacto en diversas áreas de acción en contextos universitarios. 
Un resultado relevante de esta revisión bibliográfica es que a partir de la comprensión de las bases neurobiológicas implicadas en el TDAH y los demás factores asociados es posible desarrollar estrategias neurodidácticas efectivas para los estudiantes con TDAH, que se basen en la evidencia existente en neurociencias y que incluyan todos los elementos relacionados en la expresión del trastorno.

Hace décadas, el estudio de las bases neurobiológicas del TDAH ha generado profundo interés, manteniendo un proceso constante de investigación y el uso continuo de nuevas y más precisas técnicas imagenológicas, lo que ha permitido tener un panorama general y un consenso parcial acerca de las principales alteraciones evidentes en esta condición. En esta dimensión, podemos concluir que existen diversas bases neurobiológicas que pueden funcionar tanto como causa y/o expresión del trastorno.

La neurodidáctica hoy, aparece como una alternativa para contrarrestar y aportar estrategias que permitan equilibrar estas dificultades y, asimismo, potenciar las herramientas particulares con las que podrían contar los estudiantes. Siendo la incorporación de la actividad motora, el humor, la planificación de las actividades más cortas, monitoreadas y la distribución del espacio algunos de los aportes que podrían contribuir positivamente al desempeño académico de estudiantes con TDAH.

Se concluye que el actual contexto universitario tiene aspectos que inciden negativamente en los procesos de aprendizaje de estudiantes con TDAH, porque mantiene espacios sobrepoblados, con cargas académicas excesivas, rutinas extenuantes, niveles bajos de supervisión, fragmentación del currículum y tendencia a la clase expositiva por sobre las actividades prácticas, lo que es perjudicial, precisamente para las funciones alteradas en esta condición (Olivos, 2011).

Entendiendo que el desempeño académico se ve afectado por la presencia del TDAH, las consecuencias directas que esta investigación logró recopilar tienen que ver con la vinculación existente entre las características clínicas de cuadro y un rendimiento deficitario, expresado a partir de bajos promedios, mayor deserción, cambios de carreras y mayor tiempo en completar los programas de estudio (Ortiz y Medrano, 2016).

Además, se logra concluir que existen variables propias de los alumnos con TDAH que podrían incidir indirectamente en el desempeño académico, estas serían la consecuencias psicoemocionales y sociales, alta comorbilidad con otros cuadros, cobrando especial atención la predisposición al consumo y abuso de drogas.

En esta revisión se limitaron los criterios de búsqueda para darle una metodología consistente y estructurada a la recolección de datos. Se revisaron múltiples tipos de estudios, los cuales presentaban técnicas, enfoques y principios diversos para abordar la temática, lo que hizo compleja la interpretación de los textos revisados y la compilación de manera coherente de la bibliografía. Sin embargo, eso terminó siendo una fortaleza para lograr un análisis más completo, multidisciplinario y global en este estudio.

Una de las limitaciones de este estudio es que al relacionar áreas de análisis distintas la búsqueda no necesariamente responde al cruce de estas. Por otro lado, en la comprensión del TDAH aún no existe consenso en algunos aspectos de este, lo que dificulta la homogeneidad de los estudios y sus posibles interpretaciones. 


\section{CONCLUSIONES}

Los estudiantes de educación superior con TDAH pueden tener su desempeño académico afectado por consecuencias directas o indirectas del trastorno. Esto lleva consigo desafíos específicamente en el área de la docencia, desafíos que podrían enfrentarse desde la neurodidáctica, siendo esta una herramienta que permite el desarrollo de metodologías que beneficien a todo el estudiantado.

Esta investigación permite comprender cómo el TDAH impacta en áreas del desarrollo neurocognitivo que son de vital importancia para tener un desempeño eficiente en la educación superior, siendo esencial considerar que el sistema atencional estaría alterado, y que disminuyen considerablemente las habilidades para responder a un estímulo relevante, en la atención voluntaria, en la rapidez del procesamiento de la información y en la atención selectiva. Un aspecto a tener en consideración son las fallas que se visualizan en relación a las funciones ejecutivas como la autorregulación del comportamiento, desinhibición, impulsividad, dificultades en el control de respuestas automáticas, hiperactividad y dificultades en la regulación de emociones. La neuropsicología propia del TDAH y sus comorbilidades afectan el desempeño académico de estudiantes de educación superior, por lo que generar sugerencias para las metodologías de enseñanza-aprendizaje para estudiantes universitarios con trastorno de déficits atencional se vuelve una responsabilidad para docentes y especialistas en el área, tomando en consideración las implicancias que se exponen en este artículo en los procesos de aprendizaje y vinculación social.

En esta revisión se pretende concluir la importancia que tiene conocer las características neurobiológicas, cognitiva-conductuales y destacar la relevancia de la heterogeneidad del cuadro, el contexto y el rol protagonista que podría tener el docente para fortalecer y potenciar las habilidades necesarias para lograr un aprendizaje significativo en el aula en este perfil de estudiantes.

La información concluida invita a la generación de nuevas interrogantes y promueve la investigación de otros aspectos relacionados con la temática propuesta, uno de ellos es la necesidad de identificar la real prevalencia del trastorno en estudiantes de educación superior en Chile, por otro lado, es necesario reconocer estrategias efectivas en estudiantes con TDAH y por último el reconocimiento de aspectos menos estudiados como el autoconcepto, los intereses y su relación con la educación superior.

\section{REFERENCIAS BIBLIOGRÁFICAS}

Aboitiz, F., Ossandón, T., Zamorano, F. \& Billeke, Y. P. (2012). Balance en la cuerda floja: la neurobiología del trastorno por déficit atencional e hiperactividad. Revista Médica Clínica Las Condes, 23(5), 559-565. https://doi.org/10.1016/S0716-8640(12)70350-4

Almeida Montes, L. G., Ricardo-Garcell, J., Prado Alcántara, H. y Martínez García, R. B. (2009). Alteraciones estructurales encefálicas en el trastorno por déficit de atención e hiperactividad: una actualización. Primera parte. Salud mental, 32(6), 503-512.

American Psychiatric Association. (2014). Guía de consulta de los criterios diagnósticos del DSM5®: Spanish Edition of the Desk Reference to the Diagnostic Criteria From DSM-5®. American Psychiatric Pub.

Asherson, P., Akehurst, R., Kooij, J. Smontes., Huss, M., Beusterien, K., Sasané, R., Gholizadeth, S. y Hodgkins, P. (2012). Under diagnosis of adult ADHD: cultural influences 
and societal burden. Journal of Attention Disorders, 16(5_suppl), 20S-38S. https://doi. org/10.1177/1087054711435360

Baird, A. L., Coogan, A. N., Siddiqui, A., Donev, R. M. y Thome, J. (2012). Adult attention-deficit hyperactivity disorder is associated with alterations in circadian rhythms at the behavioural, endocrine and molecular levels. Molecular psychiatry, 17(10), 988. https://doi.org/10.1038/ mp.2011.149

Barandiarán, A. A. y Samaniego, M. C. M. (2013). Televisión, clima familiar y percepción de valores en adolescentes con TDA-H y adolescentes estándar. Revista Latina de Comunicación Social, (68), 10-16. doi: 10.4185/RLCS-2013-976

Barkley, R. A., Murphy, K. R. y Fischer, M. (2010). ADHD in adults: What the science says. Guilford Press.

Bruner, M. R., Kuryluk, A. D. y Whitton, S. W. (2015). Attention-deficit/hyperactivity disorder symptom levels and romantic relationship quality in college students. Journal of American College Health, 63(2), 98-108. https://doi.org/10.1080/07448481.2014.975717

Castillo, O. R. G. (2015). Trastorno por déficit de atención e hiperactividad. Editorial El Manual Moderno.

Colomer, C., Mercader, J., Berenguer, C., Roselló, B. y Miranda, A. (2015). Percepciones de los déficits de funcionamiento ejecutivo de adultos con TDAH versus percepciones de sus familiares. Revista INFAD de Psicología. International Journal of Developmental and Educational Psychology, 2(1), 291-300.

Corbetta, M. y Shulman, G. L. (2002). Control of goal-directed and stimulus-driven attention in the brain. Nature reviews neuroscience, 3(3), 201.

Cunill, R., y Castells, X. (2015). Trastorno por déficit de atención con hiperactividad. Medicina Clínica, 144(8), 370-375. https://doi.org/10.1016/j.medcli.2014.02.025

DEMRE. (2018). Solicitud de ajustes para postulantes en situación de discapacidad. Recuperado el noviembre de 12 de 2018, de http://psu.demre.cl/inscripcion/postulantes-con-discapacidad

Fukushi, K. (2010). El nuevo alumno y el desafío de la meritocracia: análisis del cambio cultural en la educación superior chilena. Calidad en la Educación, (33). http://dx.doi.org/10.31619/caledu. n33.148

Jansen PJC, Ramos-Quiroga, J. A., Chalita, P. J., Vidal, R., Bosch, R., Palomar, G. y Prats, L. (2012). Diagnóstico y tratamiento del trastorno por déficit de atención/hiperactividad en adultos. Rev neurol, 54(Supl 1), S105-15.

Hasler, R., Perroud, N., Meziane, H. B., Herrmann, F., Prada, P., Giannakopoulos, P. y Deiber, M. P. (2016). Attention-related EEG markers in adult ADHD. Neuropsychologia, 87, 120-133. https:// doi.org/10.1016/j.neuropsychologia.2016.05.008

Kasparek, T., Theiner, P. y Filova, A. (2013). Neurobiology of ADHD From Childhood to Adulthood. Journal of Attention Disorders, 19(11),931-943. https://doi.org/10.1177\%2F1087054713505322

Kollins, S. (2009). Genética, neurobiología y neurofarmacología del trastorno por déficit de atención e hiperactividad (TDAH). Revista Española de Toxicomanías, 55, 19-28.

Kollins, S. H., English, J. S., Roley, M. E., O’Brien, B., Blair, J., Lane, S. D. \& McClernon, F. J. (2013). Effects of smoking abstinence on smoking-reinforced responding, withdrawal, and cognition in adults with and without attention deficit hyperactivity disorder. Psychopharmacology, 227(1), 19-30.

Kuriyan, A. B., Pelham, W. E., Molina, B. S., Waschbusch, D. A., Gnagy, E. M., Sibley, M. H., Babinski, D., Walther, C., Cheong, J., Yu, J. y Kent, K. M. (2013). Young adult educational and vocational outcomes of children diagnosed with ADHD. Journal of abnormal child psychology, 41(1), 27-41.

Kwon, S. J., Kim, Y. y Kwak, Y. (2018). Difficulties faced by university students with self-reported symptoms of attention-deficit hyperactivity disorder: a qualitative study. Child and adolescent psychiatry and mental health, 12(1), 12. 
López, S., Margarita, I., Rodillo, B. y Kleinsteuber, S. (2008). Neurobiología y diagnóstico del trastorno por déficit de atención. Rev. Med. Clin. Condes, 19(5), 511-524.

López-Martín, S., Albert, J., Fernández-Jaén, A. y Carretié, L. (2010). Neurociencia afectiva del TDAH: Datos existentes y direcciones futuras. Escritos de Psicología (Internet), 3(2), 17-29.

Mayor, J. G. y García, R. S. (2011). Trastorno por Déficit de atención/Hiperactividad (TDAH) Revisión ¿Hacia dónde vamos ahora. Revista Chilena de Psiquiatría y Neurología de la Infancia y la adolescencia, 22(2), 144-154.

Mellado, M. B., Martínez, L. N. y Tello, F. P. H. (2013). Procesos atencionales implicados en el Trastorno por Déficit Atencional con Hiperactividad (TDAH). Convergencia educativa, (2), 9-19.

Michalek, A. M., Watson, S. M., Ash, I., Ringleb, S. y Raymer, A. (2014). Effects of noise and audiovisual cues on speech processing in adults with and without ADHD. International journal of audiology, 53(3), 145-152 https://doi.org/10.3109/14992027.2013.866282

Montes de Oca Recio, N. y Machado Ramírez, E. F. (2011). Estrategias docentes y métodos de enseñanza-aprendizaje en la Educación Superior. Humanidades Médicas, 11(3), 475-488

Nielsen, N. P. y Wiig, E. H. (2011). AQT cognitive speed and processing efficiency differentiate adults with and without ADHD: A preliminary study. International journal of psychiatry in clinical practice, 15(3), 219-227. https://doi.org/10.3109/13651501.2011.582538

Olivos, T. M. (2011). Didáctica de la Educación Superior: nuevos desafíos en el siglo XXI. Perspectiva educacional, 50(2), 26-54.

Ortiz, S. y Jaimes, A. (2016). Trastorno por déficit de atención en la edad adulta y en universitarios. Revista de la Facultad de Medicina de la UNAM, 6-4.

Paniagua G, M. N. (2013). Neurodidáctica: Una nueva forma de hacer educación. Fides et RatioRevista de Difusión cultural y científica de la Universidad La Salle en Bolivia, 6, 72-77.

Paris, J., Bhat, V. y Thombs, B. (2015). Is Adult Attention-Deficit Hyperactivity Disorder Being Overdiagnosed? Canadian Journal of Psychiatry. Revue Canadienne de Psychiatrie, 60(7), 324 328. https://doi.org/10.1177\%2F070674371506000705

Plichta, M. M., Vasic, N., Wolf, R. C., Lesch, K. P., Brummer, D., Jacob, C., Fallgatter, A \& Grön, G. (2009). Neural hyporesponsiveness and hyperresponsiveness during immediate and delayed reward processing in adult attention-deficit/hyperactivity disorder. Biological psychiatry, 65(1), 7-14. https://doi.org/10.1016/j.biopsych.2008.07.008

Prevatt, F. y Young, J. L. (2014). Recognizing and treating attention-deficit/hyperactivity disorder in college students. Journal of College Student Psychotherapy, 28(3), 182-200. https://doi.org/10. 1080/87568225.2014.914825

Portela Sabari, A., Carbonell Naranjo, M., Hechavarría Torres, M. y Jacas García, C. (2016). Trastorno por déficit de atención e hiperactividad: algunas consideraciones sobre su etiopatogenia y tratamiento. Medisan, 20(04), 556-607.

Quintero, J., Loro, M., Jiménez, B. y Campos, N. G. (2011). Aspectos evolutivos del Trastorno por Déficit de Atención e Hiperactividad (TDAH): de los factores de riesgo al impacto socioacadémico ya la comorbilidad. Vertex. Revista Argentina de Psiquiatría, 22(96), 101-108.

Ramos, C. A. y Pérez Salas, C. P. (2015). Relación entre el modelo híbrido de las funciones ejecutivas y el trastorno por déficit de atención con hiperactividad. Psicología desde el Caribe, 32(2), 299314.

Rodillo, B. E. (2015). Trastorno por déficit de atención e hiperactividad (TDAH) en adolescentes. Revista Médica Clínica Las Condes, 26(1), 52-59. https://doi.org/10.1016/j.rmclc.2015.02.005

Rodríguez Portuguez, G. (2016). Funciones ejecutivas, rasgos de personalidad y rendimiento académico en estudiantes universitarios de ciencias de la salud (Vol. 398). Ediciones Universidad de Salamanca.

Rogers, D. C., Dittner, A. J., Rimes, K. A. \& Chalder, T. (2017). Fatigue in an adult attention deficit hyperactivity disorder population: A trans-diagnostic approach. British Journal of Clinical Psychology, 56(1), 33-52. https://doi.org/10.1111/bjc.12119 
Said N. (2013) Predicting Academic Performance: Executive Functions, Metacognition, Study Strategies, and Self-Efficacy. The 2013 WEI International Academic Conference Proceedings. Vol. $134-47$.

Salmi, J., Salmela, V., Salo, E., Mikkola, K., Leppämäki, S., Tani, P., Hokkanen, L., Laasonen, M., Numminen, J. y Alho, K. (2018). Out of focus-brain attention control deficits in adult ADHD. Brain Research.

Santander, J., Berner, J. E., Contreras, A. M. \& Gómez, T. (2013). Prevalencia de déficit atencional en estudiantes de medicina de la Pontificia Universidad Católica de Chile. Revista chilena de neuro-psiquiatría, 51(3), 169-174. http://dx.doi.org/10.4067/S0717-92272013000300002

Schoeman R., Albertyn R., de Klerk M. (2017). Adult attention-deficit hyperactivity disorder: Why should we pay attention? S Afr J Psychiatr 23, a1072. doi: 10.4102/sajpsychiatry.v23i0.1072.

Slachevsky Ch, A., Pérez, C., Silva, J. R., Ruiz-Tagle, A., Mayol, R., Muñoz-Neira, C. y NúñezHuasaf, J. (2012). Descomponiendo el síndrome de déficit atencional en el adulto: hacia un entendimiento de su heterogeneidad pronóstica. Revista médica de Chile, 140(3), 379-385. http://dx.doi.org/10.4067/S0034-98872012000300016

Strauß, M., Ulke, C., Paucke, M., Huang, J., Mauche, N., Sander, C., Stark, T y Hegerl, U. (2018). Brain arousal regulation in adults with attention-deficit/hyperactivity disorder (ADHD). Psychiatry research, 261, 102-108. https://doi.org/10.1016/j.psychres.2017.12.043

Thomas, R., Sanders, S., Doust, J., Beller, E. y Glasziou, P. (2015). Prevalence of attention-deficit/ hyperactivity disorder: a systematic review and meta-analysis. Pediatrics, peds-2014. doi: 10.1542/peds.2014-3482

Ticas, J. A. R. y Ochoa, E. R. (2010). Trastorno por Déficit de Atención e Hiperactividad (TDAH) en adultos. Rev Med Hondur, 78(4).

Valdizán, J. R. y Izaguerri-Gracia, A. C. (2009). Trastorno por déficit de atención/hiperactividad en adultos. Rev Neurol, 48(Supl 2), S95-9.

Vera Carrasco, O. (2009). Cómo escribir artículos de revisión. Revista Médica La Paz, 15(1), 63-69.

Volkow, N. D., Wang, G. J., Newcorn, J. H., Kollins, S. H., Wigal, T. L., Telang, F., Fowler, J. S., Goldstein, R. Z., Klein, N., Logan, J., Wong, C.,Swanson, J. M. (2010). Motivation deficit in ADHD is associated with dysfunction of the dopamine reward pathway. Molecular psychiatry, 16(11), 1147-54. https://doi.org/10.1038/mp.2010.97

White, H. A., y Shah, P. (2016). Scope of semantic activation and innovative thinking in college students with ADHD. Creativity Research Journal, 28(3), 275-282. https://doi.org/10.1080/104 00419.2016.1195655

Willcutt, E. G. (2012). The prevalence of DSM-IV attention-deficit/hyperactivity disorder: a metaanalytic review. Neurotherapeutics, 9(3), 490-499. doi: 10.1007/s13311-012-0135-8

York, T. T., Gibson, C., y Rankin, S. (2015). Defining and Measuring Academic Success. Practical Assessment, Research \& Evaluation, 20.

Zuluaga-Valencia, J. B., y Fandiño-Tabares, D. C. (2017). Comorbilidades asociadas al déficit de atención con hiperactividad. Revista de la Facultad de Medicina, 65(1), 61-66. http://dx. doi. org/10.15446/revfacmed.v65n1. 57031. 